\title{
A MEMS RADIO-FREQUENCY ION MOBILITY SPECTROMETER FOR CHEMICAL AGENT DETECTION
}

\author{
Raanan A. Miller, Gary A. Eiceman \\ Charles Stark Draper Laboratory, MS 37, 555 Technology Square, Cambridge, MA 02139 \\ "Department of Chemistry, New Mexico State University, Las Cruces, NM 88003
}

\begin{abstract}
A first-of-a-kind MEMS Radio-Frequency Ion Mobility Spectrometer (RF-IMS) with a miniature drift tube $\left(0.6 \mathrm{~cm}^{3}\right)$ has been fabricated and tested. The spectrometer has detection limits in the parts-per-billion and the ability to identify chemicals not resolved in conventional time-of-flight ion mobility spectrometry. Spectrometer operation with a miniature $10.6 \mathrm{eV}(\lambda=116.5 \mathrm{~nm}) \mathrm{UV}$ photo-dischargc lamp and a $100 \mu \mathrm{Ci}$ radioactive ionization sourcc has been demonstrated. The RF-IMS has been interfaced to a mass spectrometer and RF-IMS spectral peaks have been confirmed. The RF-IMS/MS configuration illustrates another use for the RFIMS as a pre-filter for atmospheric pressure chemical ionization mass spectrometry applications.
\end{abstract}

\section{INTRODUCTION}

Ion Mobility Spectrometry is the leading technology for onsite detection of chemical warfare agents, explosives, and illicit drugs [1]. It is also the technology chosen by NASA for air quality monitoring aboard the international space station [2]. IMS is inherently suitable for field operation as it utilizes a relatively simple fluidic system and operates at atmospheric pressure. It is highly sensitive, portable, has low power consumption and moderate cost.

Commercially available IMS systems are based on time-offlight (TOF), i.e., they measure the time it takes ions to travel from a shutter-gate to a detector through an inert atmosphere ( 1 to 760 Torr). The drift time is dependent on the mobility of the ion (i.e., its size, mass and charge) and is characteristic of the ion species detected [1]. The central component of the IMS is the drift tube, which ultimately determines the instrument's performance [3]. The drift tube consists of an ionization region, a shutter, a drift region and a detector. Current spectrometers use conventionally machined drift tubes (minimum size about $40 \mathrm{~cm}^{3}$ ) for ion identification, but future applications will require drift tube designs and fabrication technologies which enable even smaller, lower cost, lower power and more reproducible spectrometers. MEMS technology offers a natural path to realizing such spectrometers. Yet to-date, attempts to fabricate miniaturized MEMS IMS drift tubes have lead to spectrometers with poor sensitivity or resolution $[4,5]$.

In this work, a recently developed IMS-based technique, RFIMS, has been adopted. This method uses the non-linear mobility dependence of ions on high strength RF electric fields for ion filtering [6]. The RF-IMS technique scales down well, allowing miniaturization of IMS drift tubes through MEMS, while preserving sensitivity and resolution [7]. In this paper, we report on the fabrication and characterization of a novel MEMS RF-IMS.

\section{RF-IMS OPERATION PRINCIPLE}

Conventional time-of-flight ion mobility spectrometers (TOFIMS) operate in the low field regime where the applied field strength is less than $1000 \mathrm{~V} / \mathrm{cm}$ and the mobility is essentially constant. However, Mason and McDaniel [8] found that the mobility of an ion is field dependent and can change significantly as the field strength increases. Different ion species will have particular mobility dependencies on electric field, see Fig. 1. The RF-IMS utilizes the change in mobility between the high field and low field conditions to identify the different ion species.

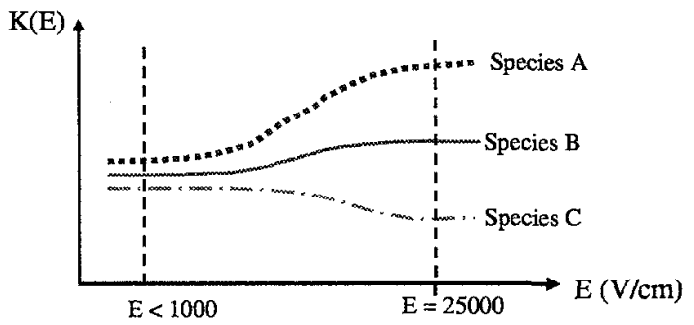

Figure 1. Mobility dependence on electric field for three hypothetical ions.

Fig. 2 illustrates the operation of the RF-IMS. As a gas sample is introduced into the spectrometer it is ionized and the ions are transported through an ion filter towards a detector by a carrier gas. The ion filter is a tunable filter which selects the ion species allowed to pass through the filter by adjusting the RF and compensation electric fields applied between the ion filter electrodes. The filtering mechanism is governed by the interaction between the ion and the net applied field which alternates between high and low electric field strengths.

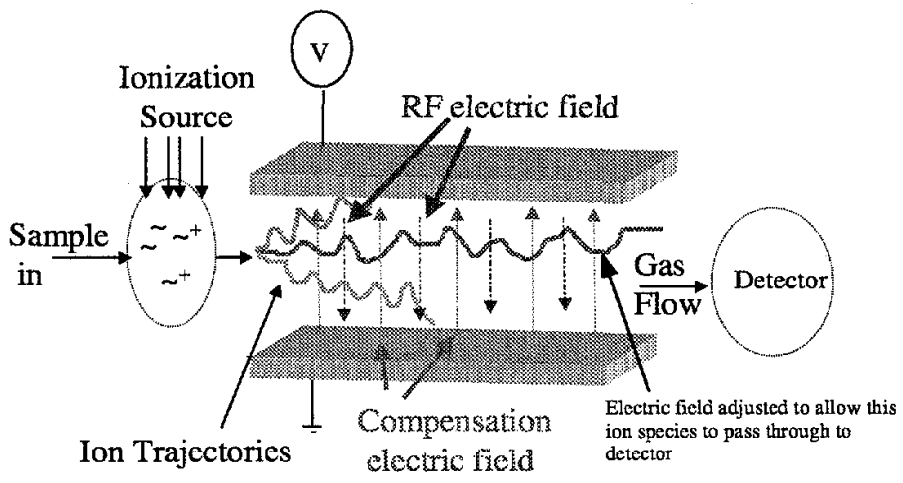

Figure 2. Schematic showing ions filtered by the $R F$ and compensation fields applied between the filter.

The conventional IMS operates with short pulses of ions (usually produced by a shutter-gate) which allow only about $1 \%$ of the ions formed in the ionization region to reach the detector [3]. Thus, the ion current is limited by the fixed number of ions contained in the ion pulse. Miniaturization of the conventional IMS therefore, can significantly decrease the number of ions available for detection. The pulse width can not be increased however, since resolution in the TOF-IMS is dictated by the width of the ion pulse. The wider the pulse, the poorer the resolution.

A significant advantage of the RF-IMS approach is that it does not require ion pulses for operation and the resolution is not dictated by the width of the ion pulse. The ions are introduced continuously into the ion filter and almost $100 \%$ of the "tuned" 
ions are passed through the filter, maintaining the high sensitivity of the device. The RF-IMS approach also avoids the complexity of generating short, spatially well confined, ion pulses. In fact, the RF-IMS approach actually benefits from miniaturization since the electric fields required to filter the ions are on the order of 10,000 $\mathrm{V} / \mathrm{cm}$. By keeping the dimensions small, aboul 500 microns, the voltages required for ion filtering are easily realizable.

Further insight into the operation principle of the RF-IMS can be gained by considering a single ion, transported by a gas stream between two parallel electrodes as shown in Fig. 3a. The ion experiences a force from the carrier gas flow ( $\mathrm{z}$-directed) which transports it through the filter. A transverse force (y-directed) produced by combining the RF electric field and a DC compensation field also acts on the ion. These fields are generated by applying voltages to the parallel plate electrodes. A (simplified) $\mathrm{RF}$ electric field waveform, Fig. $4 \mathrm{a}$, with maximum field strength $\left|E_{\max }\right|>10,000 \mathrm{~V} / \mathrm{cm}$ and minimum field strength $\left|E_{\min }\right|<<\left|E_{\max }\right|$ is used here to illustrate the ion filtering principle. The asymmetric $\mathrm{RF}$ waveform is designed such that the time average electric field is zero and

$$
\left|E_{\max }\right| t_{l}=\left|E_{\min }\right| t_{2}=\beta
$$

where $t_{l}$ is the portion of the period where the high field is applied, $t_{2}$ is the time the low field is applied, and $\beta$ is a constant corresponding to the area under-the-curve in the high field and low field portions of the waveform. The RF field causes the ions to oscillatc in a direction transverse to the carrier gas flow as they are transported down the channel by the carrier gas, Fig. 3a. The ion velocity in the $\mathrm{y}$-direction is given by [2]

$$
V_{y}=K E
$$

Here $K$ is the coefficient of ion mobility for the ion species and $E$ is the electric field intensity, in this case entirely in the y-direction. At a constant density $N$ of carrier gas the dependence of the mobility on the electric field intensity can be represented by the following expression $[7,8]$

$$
K(E)=K_{o}\left[I+\alpha_{2} E^{2}+\alpha_{4} E^{4}+. .\right]
$$

where $\alpha_{2}$ and $\alpha_{4}$ are coefficients of a series expansion, and $K_{o}$ is the mobility coefficient in a vanishingly small field [6]. As the electric field strength increases (above $5000 \mathrm{~V} / \mathrm{cm}$ ) the second and higher order terms in the series become significant and the mobility coefficient can change substantially $(10-15 \%)$ from its low field value, Fig. 1 [7]. For illustration here, it is asumed the mobility increases with increased field strength therefore $K_{1}$ (at $E_{\max }$ ) $>K_{2}$ (at $E_{\min }$ ). The ion displacement from its initial position in the y-direction is the ion velocity in the $y$-direction, $V_{y}$, multiplied by the length of time, $\Delta t$, the field is applied

$$
\Delta y_{R F}=V_{y} \Delta t
$$

In one period of the applied RF field the ion moves in both the positive and negative y-directions. By substituting equation (2) into equation (4) the average displacement of the ion over one period can be written as

$$
\Delta y_{R F}=K_{1}\left|E_{\max }\right| t_{1}-K_{2}\left|E_{\min }\right| t_{2}
$$

Using equation (1) this expression can be re-written as

$$
\Delta y_{R F}=\beta\left(K_{I}-K_{2}\right)
$$

Since $\beta$ is a constant determined by the applied RF field, the $y-$ displacement of the ion per period depends on the change in mobility of the ion between the high and low field conditions. Assuming the carrier gas only transports the ion in the z-direction, the average displacement of the ion in the $y$-direction as a function of time can be expressed as

$$
y(t)=\frac{\Delta y_{R F}}{\left(t_{1}+t_{2}\right)} t
$$

see Fig. 3b. When a low strength DC field, Fig. 4b, is applied to the ion filter electrodes $\left(\left|E_{c}\right|<\left|E_{\min }\right|<<\left|E_{\max }\right|\right)$ in a direction opposite to the average RF-induced (y-directed) motion of the ion, the trajectory of the ion species can be "straightened". This allows the ions of particular species to pass unhindered between the ion filter electrodes, Fig. 3c, while ions of all other species get deflected into the filter electrodes. The DC voltage that "tunes" the filter and produces a field which compensates for the RFinduced motion is characteristic of the ion species and is called the compensation voltage. A complete spectrum for the ions in the gas sample can be obtained by ramping or sweeping the DC compensation voltage applied to the filter.

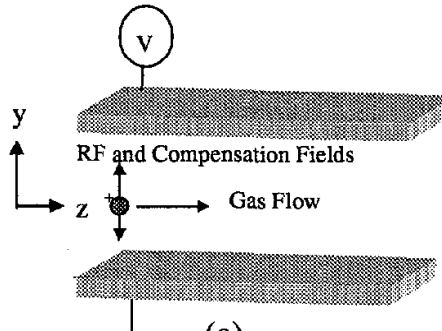

(a)

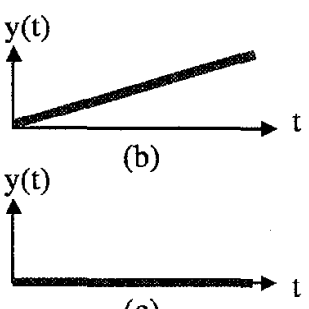

(c)
Figure 3(a) Schematic of a single positive ion located between the ion filter electrodes. (b) Average y-displacement of ion from initial position with $R F$ field applied but no compensation field applied. (c) Average y-displacement with both $R F$ and compensation fields applied.

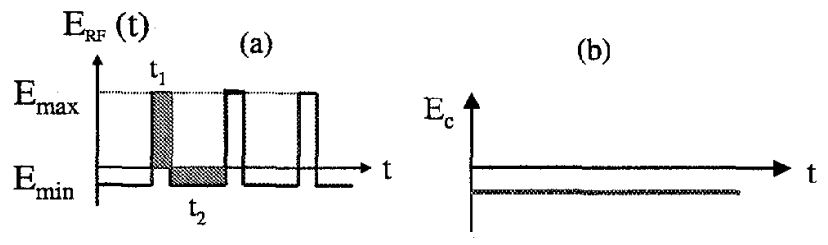

Figure 4(a) RF waveform applied to filter electrodes.(b) Compensation voltage applied to cancel out displacement produced by RF-field.

\section{DRIFT TUBE AND RF CIRCUIT DESIGN AND FABRICATION}

The fabricated MEMS RF-IMS drift tube, shown in Fig. 5, contains an ionization region, tunable ion filter electrodes, and a detector. The drift tube is fabricated from two Pyrex wafers and a heavily boron doped silicon wafer. Metal electrodes are fabricated on the top and bottom Pyrex wafers forming the ion filter, deflector and detector electrodes. The metal electrodes (1200 angstrom gold on 400 angstrom titanium) are formed on the Pyrex wafers by a sputter deposition and metal lift-off process. The Pyrex wafers are diced into rectangular pieces once the metal electrodes have been formed. Holes are drilled into the top Pyrex pieces to provide a means for the ionization sources to interact with the sample gas. The silicon wafer thickness defines the gap between the top and bottom electrodes of the ion filter. In the present method, the silicon wafer is diced into strips that are $25 \mathrm{~mm}$ long, $2 \mathrm{~mm}$ wide, and $0.5 \mathrm{~mm}$ thick. The silicon strips are aligned with the metal electrodes and anodically bonded $\left(\sim 1000 \mathrm{~V}, 350^{\circ} \mathrm{C}\right)$ to the Pyrex. Metal electrodes also make contact to these silicon strips allowing control of their potential. 


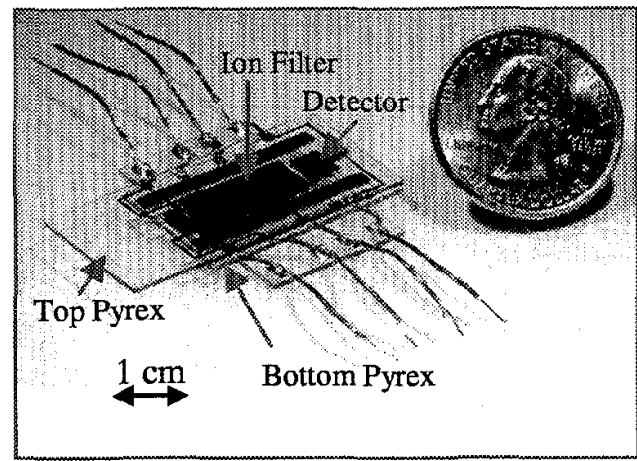

Figure 5. Photograph of a micromachined RF-IMS drift tube.

The RF field required to filter the ions is produced by a softswitched semi-resonant circuit that incorporates a flyback transformer to generate the high voltage pulses [9]. The circuit, shown in Fig. 6, provides a peak-to-peak RF voltage of 1400 volts at a frequency of $2 \mathrm{MIIz}$ with a duty cycle of about $30 \%$. In the practical implementation, the RF voltage is superimposed onto the compensation voltage.

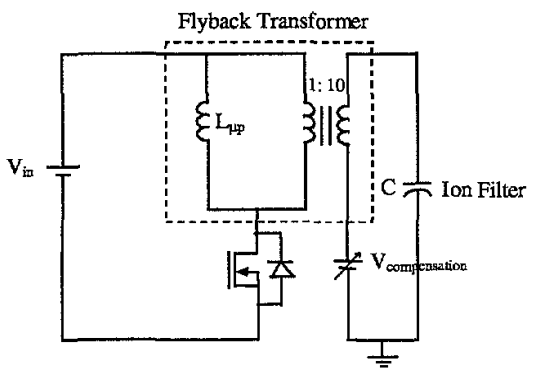

Figure 6. $R F$ waveform generator circuit uses a soft-switched semi-resonant design in order to minimize power consumption.

\section{RESULTS AND DISCUSSION}

A schematic of the experimental setup used to characterize the spectrometer is shown in Fig. 7. Sample vapors were prepared in a vapor generator using Teflon permeation cells. All supply lines following the vapor generator were heated to approximately $50^{\circ} \mathrm{C}$. For spectrometer characterization; the vapor generator was interfaced to the RF-IMS via a Teflon test fixture.

Either a miniature $10.6 \mathrm{eV}(\lambda=116.5 \mathrm{~nm})$ UV photo-discharge lamp or a $100 \mu \mathrm{Ci}$ radioactive americium source served as the ionization source for the RF-IMS. The source was positioned above the ionization region of the drift tube as shown in Fig. 7. The bottom ion filter electrode was grounded while the high voltage RF field and compensation voltages were applied to the upper filter electrode. A polynomial waveform synthesizer was used to sweep the compensation voltage and a Tektronics storage oscilloscope was used to record the signal from the detcetor amplifier and produce a spectrum for the sample gas.

Figure 8 shows the response of the RF-IMS to different concentrations of toluene vapor introduced into the spectrometer. In this experiment a UV lamp was used as the ionization source. The characteristic compensation voltage for toluene was found to be -7 volts. The peak intensity and area-under-peak depend linearly on concentration for the concentration range measured. Concentrations of toluene as low as 100 parts-per-billion have been detected. The spectrometer was also characterized with other chemicals such as acetone and benzene and each chemical exhibited its own characteristic compensation voltage peak.

The small ionization volume of the MEMS RF-IMS allows the use of low activity ionization sources such as the $100 \mu \mathrm{Ci}$

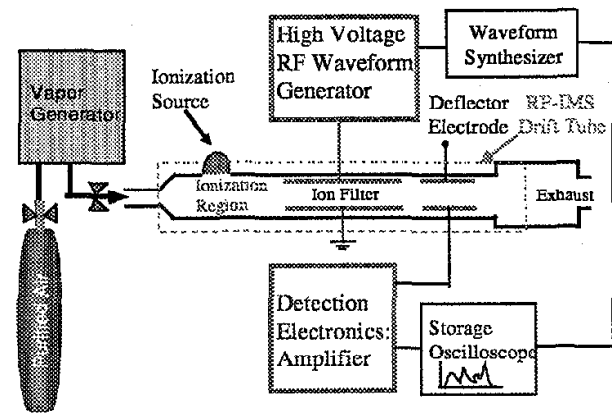

Figure 7. RF-IMS characterization setup.

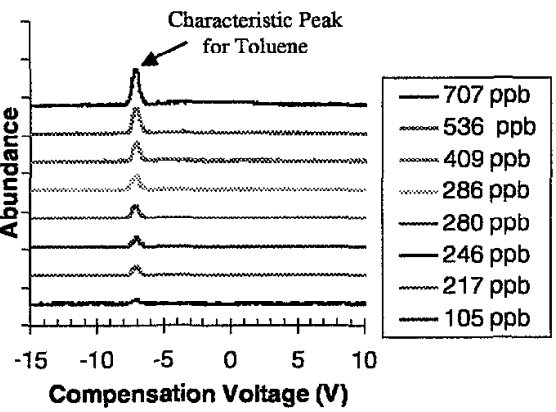

Figure 8. MEMS RF-IMS spectra for several different toluene vapor concentrations.

americium source. The americium source is the same material and activity as employed in household smoke detectors. Adopting the americium source for ionization will enable the RF-IMS to be used in many civilian applications wherc low power is important, while avoiding the extensive regulations typical of traditional TOF-IMS which use radioactive $\mathrm{Ni}^{63}$. Fig. 9 shows the spectra obtained using the americium source with dimethyl methylphosphonate (DMMPa chemical warfare agent simulant) at concentrations ranging from 300 to 90 parts-per-billion. Detection of lower concentrations were limited by the minimum vapor concentrations which the vapor generator system could deliver. The characteristic peak for DMMP is found to occur at a positive compensation voltage in contrast to toluene, acetone, and benzene which have negative compensation voltages. The small peak at about -4.5 volts, Fig. 9 , is believed to be due to an impurity present in the carrier gas which is ionized by the radioactive source. The concentration of this impurity appears to be constant throughout this experiment.

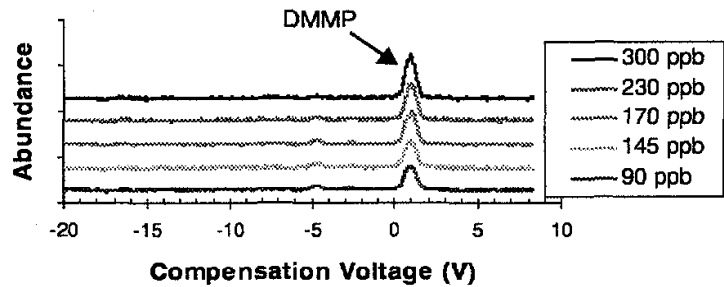

Figure 9. MEMS RF-IMS spectra for DMMP generated using a radioactive americium ionization source.

Because the RF-IMS operates in a different mobility regime compared to the traditional TOF-IMS, chemicals which are not possible to resolve in a conventional TOF-IMS can be resolved using the RF- IMS. For example, Fig. 10a shows spectra for $\mathrm{m}$ and p-xylene isomers obtained with a conventional TOF-IMS. These molecules have the same mass and very similar effective cross-sectional areas. As a result, the spectra for these two ions are virtually identical. In contrast, Fig. 10b shows the spectra obtained for these same chemicals in the MEMS RF-IMS. The peaks in this case are clearly resolved. 

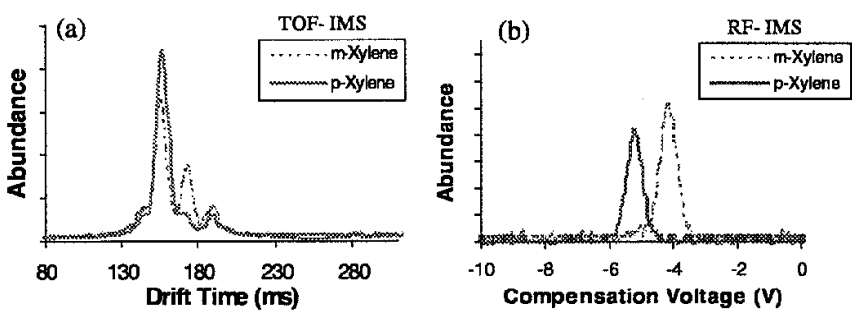

Figure 10(a)Conventional TOF-IMS spectra for $m$-xylene and $p$ xylene. (b) Spectra for $m$-xylene and p-xylene obtained with the MEMS RF-IMS.

The response of the MEMS RF-IMS to mixtures has also been investigated. Fig. 11a shows the resultant spectra for a mixture of benzene and acetone at trace concentrations ionized with a UV source. The acetone and benzene peaks are well resolved with the acetone peak occurring at a compensation voltage of -3.5 volts and the benzene peak at about -10.5 volts. Fig. $11 \mathrm{~b}$ shows spectra for benzene and acetone measured independently in a conventional TOF-IMS. It is evident that a mixture of these two chemicals would be poorly resolved in a traditional TOF-IMS.
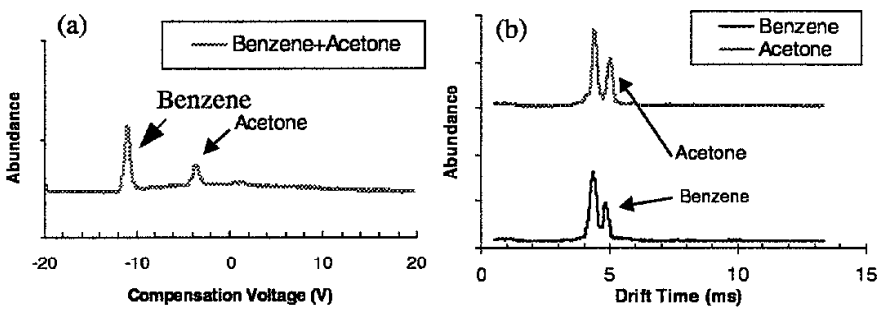

Figure 11(a) RF-IMS spectra for a benzene acetone mixture. (b) Acetone and benzene spectra measured independently in a conventional TOF-IMS.

Another exciting application for the RF-IMS, in addition to its use as a miniature detector, is as an atmospheric pressure chemical ionization (APCI) pre-filter for a mass spectrometer (MS). This pre-filtering capability should provide for enhanced discrimination of complex mixtures. The planar design and small size of the RFIMS make it easy to interface to a mass spectrometer inlet. The RF-IMS also allows control of the ions and their environment during injection into the mass spectrometer inlet. The design of the RF-IMS enables the selective injection of only ions into the mass spectrometer, while ejecting neutrals separately out the RFIMS side, Fig. 12. Both positive and negative ion species can be injected into the mass spectrometer simply by changing the polarity of the deflector electrode. The RF-IMS was interfaced to a TAGA 6000 tandem mass spectrometer as show in Fig. 12. A hole (0.7 $\mathrm{mm}$ diameter) was made in the detector electrode to facilitate the ion injection into the mass spectrometer. In addition to acting as a pre-filter for a mass spectrometer, this RF-IMS/MS configuration enables confirmation of the identities of ion species present in the RF-IMS spectral peaks.

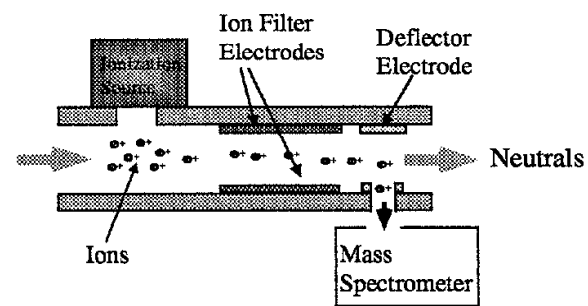

Figure 12. Schematic showing the use of the RF-IMS as a prefilter to a mass spectrometer.
Figure 13a shows the measured mass spectra with the RFIMS ion filter off. In this case all ions that are produced in the ionization region are unperturbed by the filter and can make it into the mass spectrometer. The ion species measured are acetone monomers, acetone dimers and some impurities from the carrier gas. With the RF-IMS filter on, and tuned to the characteristic compensation voltage for acetone -3.5 volts, the dominant MS peak is the acetone monomer peak all other peaks are suppressed, Fig. 13b, indicating that the RF-IMS is indeed working as a filter.
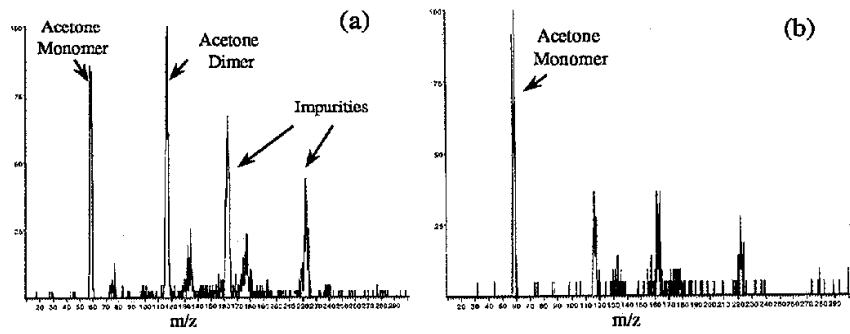

Figure 13(a) RF-IMS/MS spectra with no fields applied to the ion filter (full scale $=4.8 \times 10^{4} \mathrm{cps}$ ). (b) Spectra with the filter on and a compensation voltage of $-3.5 \mathrm{~V}$ applied (full scale $=2.2 \times 10^{4} \mathrm{cps}$ ).

\section{CONCLUSIONS}

A MEMS RF-IMS drift tube has been successfully fabricated and characterized. High spectrometer sensitivity and ability to resolve chemicals not separated in conventional TOF-IMS has been demonstrated. The MEMS RF-IMS enables the realization of miniature, low cost, high sensitivity, high reliability chemical detectors. The spectrometer has also been demonstrated as a prefilter to a mass spectrometer. The RF-IMS/MS combination will allow better resolution of complex mixtures.

\section{ACKNOWLEDGMENTS}

The Authors would like to thank the following people at Draper Laboratory: Ms. Brenda IIugh, Ms. Maria Cardoso, Mr. John Kauppinen, Dr. Jonathan Bernstein, Dr. William Kelleher and Dr. Reinhardt Willig. The authors would also like to thank Ms. Qiao Zhou of NMSU and Dr. Olga Miller of Microcosm Technologies.

\section{REFERENCES}

1. G. A. Eiceman, Z. Karpas, Ion Mobility Spectrometry, CRC Press, 1994.

2. C. S. Reese, S. J. Taraszewsky, "Near-Real-Time Analysis of Toxicologically Important Compounds Using The Volatile Organic Analyzer for the International Space Station", Proceedings of the $8^{\text {th }}$ International Conference on IMS, August 1999, Buxton, U.K.

3. Z. Karpas, "Ion Mobility in Forensic Science", Encyclopedia of Analytical Chemistry, Wiley, New York, 2000.

4. J. Xu, W. B. Whitten, and J.M. Ramsey, "Studies of Miniature Ion Mobility Spectrometer", $7^{\text {th }}$ International Conference on lon Mobility Spectrometry, Hilton Head Island, SC, 1998.

5. M. A. Butler, "Ion Trapping and Ion Mobility Spectroscopy", $7^{\text {th }}$ International Conference on Ion Mobility Spectrometry, Hilton Head Island, SC, 1998.

6. I. A. Buryakov, E. V. Krylov, E. G. Nazarov, Int. J. Mass Spectrometry and Ion Processes 128, 143- 148, 1993.

7. U. Kh Rasulev, E.G. Nazarov, V.V. Palitsin" Surface Ionization Gas-Analyzing Devices with Separation of Ions by Mobility," $4^{\text {th }}$ International Workshop on IMS, August 6-9,1995, Cambridge,U.K 8. E. A. Mason and E. W. McDaniel, Transport Properties of Ions in Gases, Wiley, New York, 1988.

9. E.V. Krylov, Instruments and Experimental Techniques, 40 (5), 628-631, 1997. 